\title{
Publisher's Note: Observation of surface superstructure induced by systematic vacancies in the topological Dirac semimetal $\mathrm{Cd}_{3} \mathrm{As}_{2}$ [Phys. Rev. B 95, 081410(R) (2017)]
}

\author{
Christopher J. Butler, Yi Tseng, Cheng-Rong Hsing, Yu-Mi Wu, Raman Sankar, Mei-Fang Wang, \\ Ching-Ming Wei, Fang-Cheng Chou, and Minn-Tsong Lin \\ (Received 2 March 2017; published 9 March 2017)
}

DOI: 10.1103/PhysRevB.95.119902

This paper was published online on 28 February 2017 with an error in a lattice parameter equation. On page 3, left-hand column, fourth paragraph, the tenth line should read as "lines), with lattice parameters $\boldsymbol{a}^{\prime}=17.9 \AA, \boldsymbol{b}^{\prime}=15.6 \AA$."

The paper has been corrected as of 3 March 2017. The text is correct in the printed version of the journal. 